\title{
Modeling National Impacts for the Building America Program*
}

\author{
Katie Coughlin and Michael McNeil \\ Energy Analysis Department \\ Environmental Energy Technologies Division \\ Lawrence Berkeley National Laboratory \\ Berkeley, CA 94720
}

March 30, 2008

\section{Contents}

1 Introduction $\quad 2$

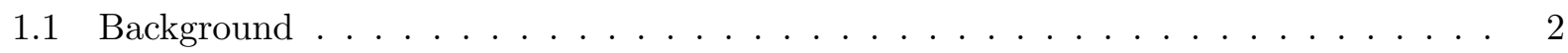

1.2 Overview of the Model Structure . . . . . . . . . . . . . . . . 4

2 Detailed Equations $\quad 6$

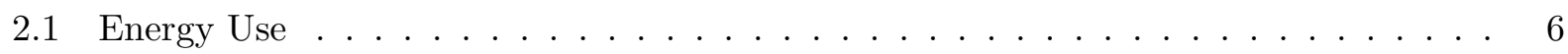

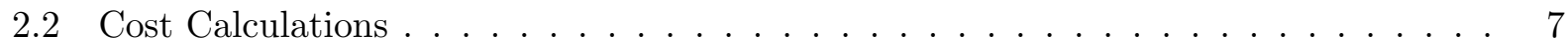

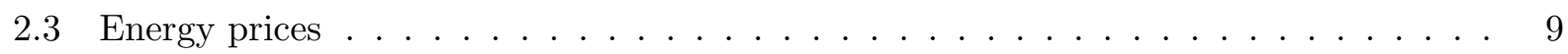

3 Modeling the builder decision $\quad 10$

3.1 Scenarios . . . . . . . . . . . . . . . . . . . . . 12

4 Total impacts $\quad 13$

$\begin{array}{ll}\text { References } & 14\end{array}$

$\begin{array}{ll}\text { A BA Model Spreadsheet } & 15\end{array}$

${ }^{*}$ This work was funded by the Assistant Secretary of Energy Efficiency and Renewable Energy of the U.S. Department of Energy under Contract No. DE-ACO3-76SF00098. 


\title{
List of Tables
}

1 Climate Zones. Each climate zone is represented using data from one city. . . . . . 5

2 Values of $\alpha_{e}$ and $\alpha_{g}$ for different values of $\alpha$, by climate zone. . . . . . . . 7

3 Reduction in potential buyers as percentage of those eligibile . . . . . . . . . . 11

4 Rising energy price factor. . . . . . . . . . . . . . . . . . . . 13

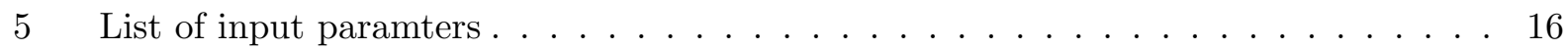

\section{List of Figures}

1 Least cost curve for the cold climate zone (Chicago) . . . . . . . . . . . . . 9

2 Log-linear plot of the least cost curve in figure 1. . . . . . . . . . . . . . . . . 10

3 Screen shot of the input selection and summary table area. . . . . . . . . . . . 17

4 Screen shot of the summary chart area. . . . . . . . . . . . . . . . . 18

\begin{abstract}
In this paper we present a model to estimate the national energy and economic impacts of the Department of Energy Building America program. The program goal is to improve energy performance in new residential construction, by working with builders to design and construct energy-efficient homes at minimal cost. The model is an adaptation of the method used to calculate the national energy savings for appliance energy efficiency standards. The main difference is that the key decision here is not the consumer decision to buy an efficient house, but rather the builder decision to offer such a house in the market. The builder decision is treated by developing a number of scenarios in which the relative importance of first costs vs. energy savings is varied.
\end{abstract}

\section{Introduction}

\subsection{Background}

The Government Performance and Results Act (GPRA) of 1993 requires all federal agencies to quantify the benefits to the nation of their programs [7]. The Department of Energy (DOE) office of Energy Efficiency and Renewable Energy (EERE) is supporting the development of a uniform methodology to be used for all of its programs, based on recommendations made in a National Academy of Sciences study published in 2001[8]. The metrics used are similar to those used to evaluate the potential benefits of DOE appliance and equipment efficiency standards [15], and include consumer energy cost savings and total source energy displaced, and the resulting reductions to carbon emissions, fuel consumption, and supply capacity additions. Programs are generally evaluated assuming that they meet their stated program goals.

For the most part EERE program impacts are estimated using a specially adapted version of the National Energy Modeling System (NEMS). NEMS is used by the Energy Information Agency 
(EIA) to produce the Annual Energy Outlook (AEO), which provides a forecast of energy supply and demand by sector and by fuel type for a period of 25 years from the publication date [11]. The forecast includes estimates of electricity and other fuel demands for residential and commercial buildings, broken down by major end use and regionally by census division. The GPRA version of NEMS uses a specially developed baseline case, which removes any EERE program activities that are explicitly accounted for in the standard AEO. A set of program cases are then defined which add back each EERE program one at a time. The analysis period depends on the program, and may exceed the AEO forecast period. If necessary, off-line calculations are used to estimate some of the impacts, which are then represented by modifying the quantities NEMS uses to build up its energy demand forecasts [1].

Building America directly impacts new housing, and indirectly impacts the rate of penetration of energy-efficient components and methods in all residential construction. NEMS represents residential energy demand as a set of end-uses (space heating, space cooling, lighting etc. ) [12]. To account for regional variation, end-use demand functions are defined for each census division. The seasonal and hourly variation of demand within a year is defined by a fixed load-shape, and the total annual demand is recalculated each year based on forecast floor space growth, efficiency trends, energy prices and other variables. NEMS accounts for technological changes to building shell characteristics and equipment efficiencies that can slow the rate of growth of annual energy use over the forecast period. Because NEMS has no way of representing an actual building, the Building America program impacts must be calculated indirectly through their effects on shell characteristics and appliance efficiency. Each of these is treated independently within NEMS. Generally, the only way to increase the rate of penetration of a measure is to lower its first cost. While this approach works fine for analyzing programs that target specific technologies, it is problematic for Building America for two reasons. First, the Building America program looks at efficiency measures from the whole-building perspective, selecting the optimal combination of features that maximize energy savings for a given level of investment. It is not necessary for every measure to be cost-effective individually, only for the whole package to satisfy a given set of cost constraints. This presumably allows for faster penetration of the more expensive measures than would otherwise occur. Second, NEMS effectively treats the selection of all new housing features as consumer decisions. In reality (particularly for shell measures) it is usually builders who decide whether new technologies will or will not be used in construction. While this decision is clearly influenced by the builders' perception of consumer preferences, other factors impact the decision. Understanding and influencing these other factors is an important part of the Building America research program.

In this project we develop an alternative method to estimate Building America program impacts, similar to that used in the national impacts analysis for DOE appliance efficiency standards [15]. While this first version of the model is fairly simple, it has the advantage that the real structure of the Building America program is represented directly. For this reason, the relationship between program goals and program impacts is much more transparent, and the effect of changing background assumptions or program targets can be examined in a straightforward manner. 


\subsection{Overview of the Model Structure}

In this section we present a conceptual overview of how the model works. More detailed discussion, including equations and some input data, are presented in Section 2. The builder decision scenarios are discussed in Section 3, and the total impacts estimates are presented in Section 4. A brief guide to the spreadsheet itself is provided in the Appendix.

The model assumes that the Building America (BA) program does not affect either the supply of or demand for housing. Builders develop their own estimates of future housing demand and act accordingly, and there is no reason to expect this activity to be altered by the existence of efficiency programs. Because program participation is voluntary, if the proposed efficiency measures are seen as too costly or difficult, builders simply won't use them. If efficient houses do get built, we assume this is a rational decision on the part of the builder, which implies that they are as likely to sell as the baseline units. By this reasoning, there is no need to set up a detailed housing demand model to estimate program impacts. Instead, the focus is on determining which of a range of technically feasible energy efficient designs will actually be seen in the market.

The approach used here consists of three steps:

1. Estimate, for each year of the analysis period, the fraction of new housing stock that is more energy efficient due to the activities of the BA program.

2. Estimate the average energy savings per BA home as a percentage of total source energy use.

3. Total up the energy savings and net dollar benefits over the analysis period.

The fraction of new housing stock affected by the BA program is the product of the level of direct participation by builders and the proportion of units they build that are more efficient than the norm. In the current version of the model, this fraction is a user input, which we call $\beta$. In general we expect $\beta$ to increase over time, however as the GPRA analysis looks at the future benefits of the current year's activity, in the current model we use a fixed value. The potential impacts of increased participation can be easily estimated by changing $\beta$.

To estimate the energy savings per new home, we combine the engineering estimate of potential savings with a simple builder decision model that attempts to predict the efficiency of designs that will actually get built. The least-cost curve generated by the BA engineering analysis [3] is used to quantify the level of total source energy savings achievable as a function of first cost. This curve defines the unique combination of measures that provide maximal energy savings at a given level of investment. For convenience, the additional first cost is amortized and represented as an increment to the annual mortgage payment. To estimate the savings levels that may be seen in the market, we define three scenarios which roughly represent low, medium and high sensitivity to energy prices on the part of buyers, which we label pessimistic, moderate and optimistic respectively. The scenario

places a limit on the increase in first cost that is acceptable to builders, which in turn determines the energy savings level that can be achieved. 


\begin{tabular}{|l|l|}
\hline Climate Zone & City \\
\hline hot-humid & Houston \\
mixed-humid & Atlanta \\
hot-dry & Phoenix \\
mixed-dry & Phoenix \\
marine & San Francisco \\
cold & Chicago \\
\hline
\end{tabular}

Table 1: Climate Zones. Each climate zone is represented using data from one city.

The energy savings are computed relative to a baseline defined by the AEO housing forecast and an estimate of average annual household energy use. The AEO provides a forecast of new housing floorspace by census division, which is converted to a fixed number of typical new homes. Characteristics of these new homes are taken from the BA prototype analysis [3, 6], which provides annual total gas, electricity and source energy consumption. The model calculates the energy savings per home as a percent of the annual source energy consumption, which varies by climate zone (climate zones are defined in Table 1). The AEO data is converted from census divisions to climate zones using population weighting. Both BA climate regions and census divisions are defined at the county level, so each county can be assigned to both a census division $c$ and a climate zone $r$. Let $N(r, c)$ be the total population in all counties that are in census division $c$ and climate region $r$. To convert a quantity $\tilde{X}(c)$ defined over census divisions to an equivalent quantity $X(r)$ defined over climate zones we use the formula

$$
X(r)=\frac{\sum_{c} N(r, c) \tilde{X}(c)}{\sum_{c} N(r, c)}
$$

Given an estimate of the number of units affected, and the savings per unit, the last step of the model is to construct the sum over all units for the entire analysis period. The accounting requires that we keep track of both the analysis year $y$ and the vintage $v$ (or year of construction) of a given unit. Here $v$ and $y$ are used to distinguish between variables that depend only on when a unit is built vs. those that are truly dynamic over the analysis period. For example, the annual energy consumption depends on the house design and therefore on the vintage $v$, while energy prices vary over each year of the analysis and therefore depend on $y$. In each analysis year $y$, the savings from new housing in the current year are added to cumulative savings from units built in previous years. The model calculates both the net dollar benefits to the homeowner, and the total source energy savings. The analysis period is defined as 2005 to 2030. 


\section{Detailed Equations}

\section{$2.1 \quad$ Energy Use}

BA program targets are defined in terms of source energy use, which is an appropriate metric for general societal benefits. Cost savings from the homeowner perspective depend on site energy use, so both site and source energy must be calculated in the model. Only gas and electricity are considered here. For natural gas, site and source energy are about the same (there are some delivery losses), while for electricity the source energy in equivalent units is roughly three times the amount of site energy consumed. For reference, the conversion equation used in the BA least-cost analysis is

$$
\text { Source MBtu }=\mathrm{kwh} \cdot 3.412 \cdot H_{e} / 1000+\text { therms } \cdot H_{g} / 10,
$$

where kwh is the site electricity use, therms the site gas use, and $H_{e}$ and $H_{g}$ are dimensionless site-to-source conversion factors [6]. The model uses conversion factors taken from the AEO [11]. These vary regionally, and with the year of the analysis period.

The annual on-site energy use per housing unit is defined as a function of vintage $v$, climate zone $r$ and fuel type. For the baseline unit we define:

- $E_{0}(v, r)$ is the annual baseline electricity consumption

- $G_{0}(v, r)$ is the annual baseline natural gas consumption

- $h_{e}(y, r)$ is the site-to-source conversion factor for electricity

- $h_{g}(y, r)$ is the site-to-source conversion factor for gas

- $\Gamma_{0}(y, v, r)$ is the source energy consumption

Site and source energy consumption are related through the equation

$$
\Gamma_{0}(y, v, r)=h_{g}(y, r) G_{0}(v, r)+h_{e}(y, r) E_{0}(v, r) .
$$

Note that the annual source energy use can change over time due to changes in the conversion efficiencies.

The electricity, gas and source energy use for a BA home of vintage $v$ are denoted $E(v, r)$, $G(v, r)$ and $\Gamma(y, v, r)$ respectively. These three quantities also satisfy equation (3).

To represent the energy savings of BA homes relative to the baseline, we use the following variables:

- $\alpha_{e}(v, r)$ is the percent savings for electricity consumption

- $\alpha_{g}(v, r)$ is the percent savings for natural gas consumption

- $\alpha(v, r)$ is the percent source energy savings 


\begin{tabular}{|l|l|c|c|c|c|c|}
\hline $\begin{array}{l}\text { Percent Source } \\
\text { Energy Savings }\end{array}$ & & Chicago & Houston & Phoenix & SF & Atlanta \\
\hline$\alpha=0.3$ & $\alpha_{e}$ & 0.31 & 0.36 & 0.27 & 0.31 & 0.23 \\
& $\alpha_{g}$ & 0.29 & 0.09 & 0.50 & 0.29 & 0.44 \\
\hline$\alpha=0.4$ & $\alpha_{e}$ & 0.35 & 0.40 & 0.42 & 0.31 & 0.35 \\
& $\alpha_{g}$ & 0.45 & 0.41 & 0.29 & 0.57 & 0.50 \\
\hline$\alpha=0.5$ & $\alpha_{e}$ & 0.37 & 0.44 & 0.49 & 0.34 & 0.36 \\
& $\alpha_{g}$ & 0.63 & 0.73 & 0.57 & 0.80 & 0.76 \\
\hline
\end{tabular}

Table 2: Values of $\alpha_{e}$ and $\alpha_{g}$ for different values of $\alpha$, by climate zone.

By definition, the electricity, gas and source energy use for the BA unit satisfy the equations:

$$
\begin{aligned}
& E_{0}(v, r)-E(v, r)=\alpha_{e}(v, r) E_{0}(v, r), \\
& G_{0}(v, r)-G(v, r)=\alpha_{g}(v, r) G_{0}(v, r),
\end{aligned}
$$

and

$$
\Gamma_{0}(y, v, r)-\Gamma(y, v, r)=\alpha(v, r) \Gamma_{0}(y, v, r) .
$$

The energy savings are also related through the expression

$$
\alpha \Gamma_{0}=\alpha_{e} h_{e} E_{0}+\alpha_{g} h_{g} G_{0} .
$$

The source energy savings $\alpha$ is a weighted average of the electricity and gas savings, with weights given by the relative importance of these two fuels to the total baseline source energy consumption.

Given the BA least-cost curve and a value for the initial investment, the source energy savings level $\alpha$ is determined directly. Although $\alpha_{e}$ and $\alpha_{g}$ are constrained by equation (7), more information is needed to determine their values. In principle, the relative portion of source energy savings to be allocated to each fuel type can be determined directly from the least-cost analysis. It will vary as a function of $\alpha$, as different sets of measures are used to achieve different levels of savings. In practice, it is non-trivial to generate values of $\alpha_{e}$ and $\alpha_{g}$ for each point on the least-cost curve. For the moment, the model uses representative values of $\alpha_{e}$ and $\alpha_{g}$ derived from the BA engineering analysis [4]. These are shown in Table 2.

\subsection{Cost Calculations}

The benefit to a homeowner of purchasing a BA home is the net of the increase to the annual mortgage payment and the decrease in annual energy costs. In this section we show how to calculate both sets of costs. We refer to the sum of annual mortgage payments and energy costs as the total ownership cost. We ignore other costs such as taxes as these do not affect the outcome of the analysis.

The following variables define a baseline home: 
- $P_{0}(y, r)$ is the average purchase price for a single-family dwelling in region $r$ and year $y$.

- $z(y)$ is the average mortgage rate in year $y$, here assumed to be $6 \%$ real.

- $T(y)$ is the average mortgage term in year $y$, here assumed to be 30 years.

From these, the annual mortgage payment can be determined from a standard formula $M_{0}=$ $f\left(z, T, P_{0}\right)$.

We define $Q$ to be the additional up-front cost associated with buying an energy-efficient BA home. For the moment, the value of $Q$ will be treated as an independent variable. The amortized value of this additional cost is defined as $q$ and is determined from the identity

$$
q \equiv f\left(z, T, P_{0}+Q\right)-f\left(z, T, P_{0}\right)=Q \cdot C(z, T)
$$

Here $C$ is a constant that depends only on the mortgage rate and term. All quantities depend implicitly on the year of purchase, assumed equal to the vintage, and the region.

As noted above, given $q$, the technically optimal level of source energy savings $\alpha$ is determined by the least cost curve, which will vary with vintage and climate zone. The site energy savings can then be inferred using Table 2. Formally, the model only requires that $\alpha$ be available as a mathematically well-defined function of $q, v$ and $r$, so really any rule could be used to make this association. The curve relating costs to savings is generally nonlinear, with costs rising more rapidly as $\alpha$ increases. An example curve for Chicago is shown in Figure 1 [2]. For values of $\alpha \geq 0.10$ this curve is well-fit by an exponential, as is also illustrated in Figure 2. ${ }^{1}$ We therefore assume the general approximation

$$
\alpha(q, v, r)=A \exp (B q), \quad q \geq \varepsilon>0 .
$$

The coefficients $A$ and $B$ depend on the design choices made and will therefore vary with climate zone $r$. To model the learning impacts of the BA program, the model also allows $A$ and $B$ to vary with vintage $v$. Essentially, the program lowers the cost required to reach a given level of energy savings. This type of effect is typically modeled using a simple learning curve [9].

The learning curve assumes that the cost of producing a given technology decreases with the total number of units produced, in the relationship cost $\propto$ quantity ${ }^{-s}$. The exponent $s$ is known as the learning parameter. In a review of appliance efficiency improvements, Newell [9] found values of $s$ on the order of 0.2-0.4. To represent learning effects in distributed generation technologies, NEMS uses values of $s$ of 0.15-0.2 [12]. The annual decrease in cost that results from the learning effect depends on $s$ and on the number of units produced each year relative to cumulative production. In a study of residential appliance efficiency, using Newell's data for the learning parameter and shipments data to determine production quantities, the annual cost decrease for a fixed efficiency level was found to be $1 \%-2 \%$ per year [10].

\footnotetext{
${ }^{1}$ The exponential form cannot be valid as $\alpha \rightarrow 0$, however these low values are of no practical interest in the model.
} 


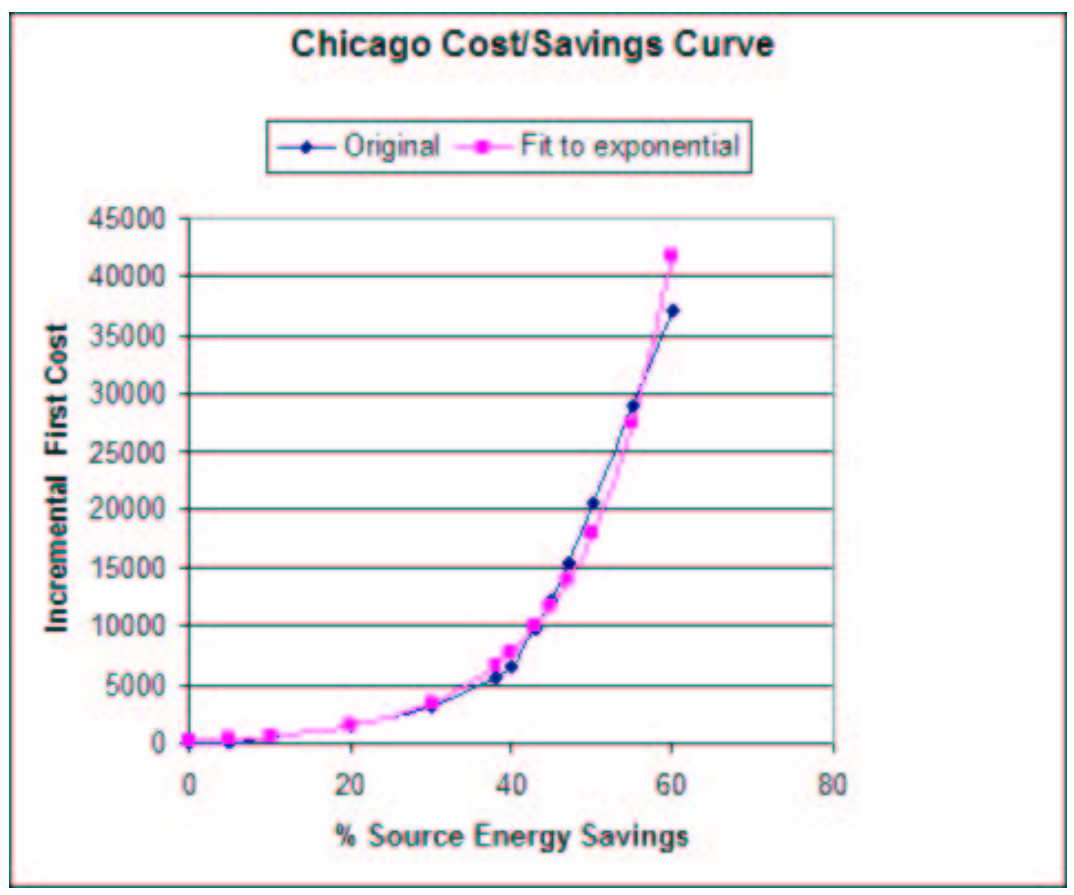

Figure 1: Least cost curve for the cold climate zone (Chicago).

The learning effect is implemented in the model by modifying the constant $A$ as follows:

$$
A(v, r)=\frac{A_{0}(r)}{(1+s)^{\left(v-y_{0}\right)}} .
$$

Here $y_{0}$ is the first year of the analysis period and $s$ is the annual percentage cost decrease mentioned above. The value of $s$ can be set as a user input, with a default value of $1.5 \%$. $A_{0}$ is determined from the curve fit illustrated in figures 1 and 2 . The constant $A$ sets the overall level of the cost curve, while $B$ determines the rate of increase of cost with savings. In principle $B$ should also vary over time due to learning, however for now the data is not sufficient to warrant this level of detail in the model. $B$ therefore depends only on region. As with the cost-savings curve, formally the model only requires that the time dependence of $A$ and $B$ be defined somehow. If desired, more elaborate models of the learning effect could be used and easily linked to the impacts model.

\subsection{Energy prices}

The AEO provides forecasts of energy prices under different economic growth scenarios. We define the regional average electricity price to be $x_{e}(y, r)$ and the natural gas price to be $x_{g}(y, r)$. In the 


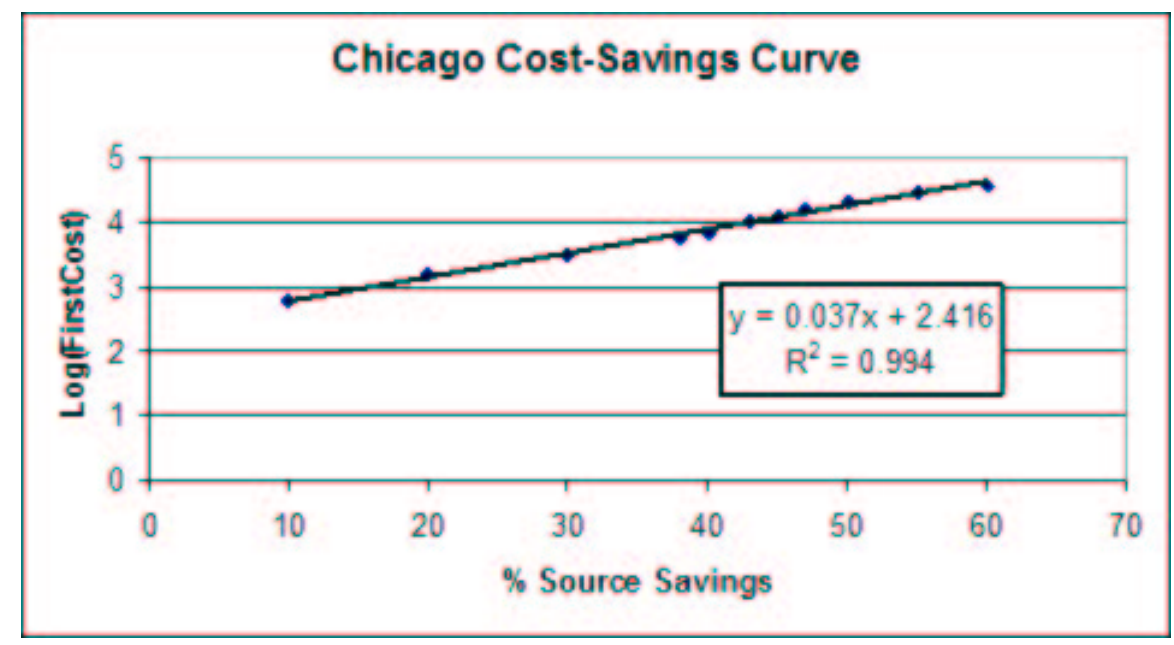

Figure 2: Log-linear plot of the least cost curve in figure 1.

base case, total annual household energy expenditures are

$$
X_{0}(y, v, r)=x_{e}(y, r) E_{0}(v, r)+x_{g}(y, r) G_{0}(v, r) .
$$

Typically electricity expenditures are somewhat higher than gas expenditures. Based on RECS 2001 [13], the annual average household expenditures are about $\$ 940$ for electricity and $\$ 700$ for natural gas. For the BA home defined by percent savings $\left(\alpha_{e}, \alpha_{g}\right)$, the annual energy cost savings are

$$
\delta X(y, v, r)=x_{e}(y, r) \alpha_{e}(v, r) E_{0}(v, r)+x_{g}(y, r) \alpha_{g}(v, r) G_{0}(v, r) .
$$

Note that the BA least-cost curves are developed assuming constant real energy prices. Depending on whether energy prices rise and by how much, the actual household savings $\delta X$ may turn out to be significantly higher, creating an additional benefit to homeowners.

\section{Modeling the builder decision}

We begin this section with a brief overview of housing price sensitivity, which provides a framework for evaluating the importance of price increases. The NAHB has published an analysis relating affordability to purchase price for over 300 metropolitan areas [5]. Their data include median house price and the income required to qualify for a typical mortgage in each area, and an estimate of the U.S. household income distribution function. They calculate the number of potential buyers that would be eliminated from the market by a given price increase by looking at the change in the number of households with income sufficient to qualify for the appropriate mortgage. Conceptually, 


\begin{tabular}{|c|c|r|r|r|r|r|}
\hline Price & Qualifying households & \multicolumn{4}{|c|}{ \% Eliminated by x\% Price Increase } \\
\hline$(1000 \$)$ & (millions) & $1 \%$ & $2 \%$ & $3 \%$ & $4 \%$ & $5 \%$ \\
\hline \hline 100 & 79.0 & $0.5 \%$ & $0.9 \%$ & $1.4 \%$ & $1.9 \%$ & $2.3 \%$ \\
150 & 61.4 & $0.8 \%$ & $1.6 \%$ & $2.4 \%$ & $3.2 \%$ & $4.0 \%$ \\
200 & 46.4 & $1.1 \%$ & $2.3 \%$ & $3.4 \%$ & $4.4 \%$ & $5.4 \%$ \\
250 & 34.6 & $1.3 \%$ & $2.6 \%$ & $4.0 \%$ & $5.3 \%$ & $6.6 \%$ \\
300 & 25.5 & $2.1 \%$ & $4.2 \%$ & $5.7 \%$ & $7.0 \%$ & $8.2 \%$ \\
350 & 19.8 & $1.8 \%$ & $3.6 \%$ & $5.4 \%$ & $7.2 \%$ & $9.0 \%$ \\
400 & 14.7 & $2.7 \%$ & $5.4 \%$ & $8.2 \%$ & $10.5 \%$ & $11.9 \%$ \\
450 & 11.5 & $2.0 \%$ & $3.9 \%$ & $5.9 \%$ & $7.8 \%$ & $9.7 \%$ \\
\hline
\end{tabular}

Table 3: Reduction in potential buyers as percentage of those eligible

if $P_{0}$ is the house price, $I\left(P_{0}\right)$ the annual income required to qualify for a mortgage, and $N(I)$ the number of households with income greater than or equal to $I$, then under a price increase $P_{0} \rightarrow P$, the number of households potentially priced out of the market is $N(I(P))-N\left(I\left(P_{0}\right)\right)$.

We have calculated the number of households eliminated in this way, as a function of house price $P_{0}$ and of the price increase expressed as a percentage of $P_{0}$. The results are displayed in Table 3 , with the number of households expressed as a percentage of those who qualify at price $P_{0}$. The number of households priced out is roughly linear in the size of the price increase, with households eliminated more rapidly in the high price range. For example, a $4 \%$ increase on a $\$ 100,000$ home would potentially eliminate $1.9 \%$ of qualified buyers, with 79.0 million households qualifying at this price. A $2 \%$ increase on a $\$ 200,000$ home could eliminate $2.3 \%$ of the 46.4 households that qualify for a mortgage at this price. Given the wide variety of mortgage options available in the market, these numbers may not reflect what would happen in reality. However, they are useful as a guide to builder perceptions.

If the decision to purchase an efficient home were purely a consumer choice, standard economic analysis tells us to look at the change in total ownership cost as a guide to rational consumer behavior. In year $y$, the net cost difference between a BA and a baseline unit of vintage $v$ is

$$
\Delta(y, v, r)=\delta X(y, v, r)-q(v, r) .
$$

Positive values of $\Delta$ represent net savings to the homeowner. Typically, consumer decision models heavily discount future savings, implying that people are most sensitive to the value of $\Delta$ in the year of purchase and possibly a few years beyond. Theoretically, if $\Delta>0$ efficiency should sell well.

The housing market differs from other markets because houses are expensive and immobile. Appliance buyers have choices because equipment manufacturers can market several lines of product with differing features and prices, and determine by experiment which are the most profitable. In contrast, the number of houses that can be produced is limited by the available land. The builder's 
investment per individual unit is orders of magnitude larger for houses than for appliances, so it is much more important to make sufficient profit on the sale of each unit. Builders are thus more limited in the range of products they are willing to offer, and the consumer choice is strongly constrained by the builder's design decisions. This may be one reason why the levels of energy savings achieved in the market are lower than what is technically feasible at neutral cost.

Within these constraints, consumer preferences still play a role in the builder decisions. If buyers are concerned about increasing energy prices, or interested in efficiency for other reasons, they may be willing to pay a premium for efficient homes. Conversely, if buyers don't seem to care about energy issues, the BA program needs to create other incentives to builder participation, for example innovations that lower construction costs, or provide other quality improvements along with efficiency. The builder finances the initial investment that leads to energy savings, and needs to recover the additional cost in the selling price. They may have to absorb some fraction of the price increase in order to sell the house. This additional cost may be small relative to the price of the house, but still significant relative to the total unit profit. Builders' perception of this financial risk places a practical limit on the additional costs they are willing to finance, and thus on the level of energy savings achievable.

Understanding these issues and overcoming diverse market barriers is a big part of the BA program. For modeling purposes, we roll all these complexities into the definition of a few numeric parameters. One is the fraction of units that are modified somehow, which is discussed in the next section. The other input we need is a rule for determining the level of source energy savings that might be seen in practice. While this depends on many factors, it is straightforward to set up a few scenarios that allow us to quantitatively distinguish between different hypotheses. Below we define three scenarios which correspond to low, medium, and high tolerance for first cost increases.

\subsection{Scenarios}

Scenario 1: Pessimistic If builders think buyers don't care about energy cost savings, then either the price increase $q$ needs to be justified by other features, such as improved comfort or advanced technology, or efficiency improvements need to be made at zero net cost. Only the latter scenario is currently modeled. Its assumed that some level of energy savings is possible without a significant price increase, with the precise value set as a user input.

Scenario 2: Moderate This scenario assumes that buyers are concerned about energy use and want to maximize efficiency, but do not anticipate rising prices. It also implicitly assumes that something like an Energy Efficiency Mortgage is available, so that energy cost savings count as income toward the mortgage and the price increase $q$ can effectively be absorbed with no change to total ownership cost. To implement the scenario, we use the practical assumption that designs are chosen to maximize the ownership cost savings. These designs correspond to the minimum on the least cost curve.

Scenario 3: Optimistic This scenario also assumes that buyers want to maximize efficiency, and in addition that they care about future energy price increases. Formally, it is similar to the 


\begin{tabular}{|c||c|c|c|c|c|}
\hline$p$ & $\mathrm{n}=1$ & $\mathrm{n}=2$ & $\mathrm{n}=3$ & $\mathrm{n}=4$ & $\mathrm{n}=5$ \\
\hline $1 \%$ & 1.01 & 1.02 & 1.03 & 1.04 & 1.05 \\
$2 \%$ & 1.02 & 1.04 & 1.06 & 1.08 & 1.10 \\
$3 \%$ & 1.03 & 1.06 & 1.09 & 1.13 & 1.16 \\
$4 \%$ & 1.04 & 1.08 & 1.12 & 1.17 & 1.22 \\
$5 \%$ & 1.05 & 1.10 & 1.16 & 1.22 & 1.28 \\
\hline
\end{tabular}

Table 4: Rising energy price factor, based on an annual percentage rate of increase $p$ and number of years in the time horizon $n$.

medium risk case, but with the energy cost savings multiplied by a rising energy price factor. This factor depends on the assumed rate of future price increases and the consumer time horizon over which these price increases are important. If $p$ is the annual percentage rate of increase, and $n$ the length of the time horizon in years, then the price factor is $(1+p)^{n}$. Some representative values, for varying $p$ and $n$, are shown in table 4 . The actual value to use is set as a user input. Note that this type of adjustment to the total ownership cost formula could also be used to model financial incentive programs, such as tax credits or rebates, or situations where buyers are willing to pay a premium for whatever reason.

\section{Total impacts}

To calculate the cumulative impacts, the model assumes that new housing can be characterized in each region by a typical prototype house, with the total number of units built determined by the AEO floorspace forecast and the typical house size. The total number of units built in year $y=v$ is denoted $n(v, r)$. The annual energy consumption per house is given by the BA prototype analysis. This can be compared for reference purposes with the annual average residential consumption output by NEMS.

The fraction of new housing units impacted by the BA program is defined the product of the market share of direct program partners times the fraction of each partner's new housing that is modified. Currently, BA partners include about 470 industrial builders, accounting for about half of new residential construction [14], for a market share of 0.5. The fraction of these companies' housing that is affected by the program is not known precisely, but is probably on the order of $10 \%$. We define the factor $\beta$ as the fraction of all new housing that is affected by the BA program, so with these defaults $\beta=0.50 \times 0.10=0.05$. Currently $\beta$ is held constant over the analysis period. As BA-encouraged building practices become more widespread, we expect $\beta$ to increase. For generality, the vintage-dependence of $\beta$ is retained in the equations below. 
The cumulative source energy savings in year $y$ due to the BA program are

$$
T E(y)=\sum_{v=y_{0}}^{y} \sum_{r} \beta(v) \alpha(v, r) n(v, r) \Gamma_{0}(y, v, r) .
$$

The undiscounted cumulative dollar costs are

$$
T Q(y)=\sum_{v=y_{0}}^{y} \sum_{r} \beta(v) \alpha(v, r) n(v, r) q(v, r),
$$

and the undiscounted cumulative dollar benefits are

$$
T X(y)=\sum_{v=y_{0}}^{y} \sum_{r} \beta(v) \alpha(v, r) n(v, r) \delta X(y, v, r) .
$$

The net dollar benefits $(T X-T Q)$ will depend both on the scenario chosen and on the energy price forecast used. The BA least-cost curves are developed assuming constant real energy prices. For the pessimistic scenario, while it has the lowest energy benefits, there will always be a dollar benefit to the homeowner. In the moderate scenario, because costs and benefits just balance, the net dollar benefit depends on the energy price forecast. If real prices rise, there will be a benefit. In the optimistic scenario, some increase in energy prices has been priced into the house, so the dollar benefits will be smaller and could even be negative if the expected energy price increase greatly exceeds the forecast. This scenario has the largest societal energy savings.

Price forecasting is a highly uncertain activity. The AEO typically includes several forecasts, and a variety of other public and private entities produce their own analyses. The model currently implements the three standard scenarios output by AEO, corresponding to low, medium and high economic growth. The economic scenario also affects the rate of increase of residential floorspace.

The model has been set up so that the user sees all the adjustable inputs and the primary outputs on the same page. This makes it easy to see the effect of changing assumptions on the most important program metrics. A more detailed discussion of the spreadsheet implementation of the model is provided in the Appendix.

\section{References}

[1] Anderson, D. M., D. B. Belzer, K. A. Cort, J. A. Dirks, D. B. Elliott, D. J. Hostick \& M. J. Scott 2003. Methodological Framework for Analysis of GPRA Metrics: Application to FY04 Projects in BT and WIP. Pacific Northwest National Laboratory, PNNL-14231.

[2] Anderson, Ren 2006. Personal communication.

[3] Anderson, R. \& C. Christensen 2004. Residential Systems Options Analysis and Development Plans. Presentation, FY05 Building America Planning Meeting. 
[4] Anderson, R., C. Christensen, G. Barker, S. Horowitz, A. Courtney, T. Givler \& Kendra Tupper 2004. Analysis of System Strategies Targeting Near-Term Building America EnergyPerformance Goals for New Single-Family Homes. National Renewable Energy Laboratory, NREL/TP-550-36920.

[5] Emrath, Paul 2005. Interest Rates and House Prices: the "Priced Out" Effect. National Association of Home Builders (NAHB) Housing Policy Focus March 2005, www.nahb.org/generic.aspx?sectionID $=734 \&$ genericContentID $=37153$.

[6] Hendron, R., R. Anderson, R. Judkoff, C. Christensen, M. Eastment, P. Norton, P. Reeves \& E. Hancock 2004. Building America Performance Analysis Procedures: Revision 1. National Renewable Energy Laboratory, NREL/TP-550-35567.

[7] National Renewable Energy Laboratory 2005. Projected Benefits of Federal Energy Efficiency and Renewable Energy Program. FY2006 Budget Request. NREL/TP-620-37931.

[8] National Research Council 2001. Energy Research at DOE: Was it Worth it? Energy Efficiency and Fossil Energy Research 1978 to 2000. www.nap.edu/books/0309074487/html.

[9] Newell, Richard 2000. Incorporation of Technological Learning into NEMS Building Modules. Prepared for the Energy Information Agency.

[10] Rosenquist, Greg, Michael McNeil, Maithili Iyer, Steve Myers \& Jim McMahon 2004. Energy Efficiency Standards and Codes for Residential/Commercial Buildings: Additional Opportunities. Report prepared for the National Commission on Energy Policy LBID-2533.

[11] US Department of Energy, Energy Information Agency, Annual Energy Outlook (AEO) 2005. DOE/EIA-0383(2005).

[12] US Department of Energy, Energy Information Agency 2005. Model Documentation Report: Commercial Sector Demand Module of the National Energy Modeling System. DOE/EIAM066(2005).

[13] US Department of Energy, Energy Information Agency, Residential Energy Consumption Survey 2001 (RECS) 2005. www.eia.doe.gov/emeu/recs/contents.html.

[14] US Department of Energy, Office of Energy Efficiency and Renewable Energy 2004. Building America Puts Residential Research Results To Work. www.eere.energy.gov/buildings/building_america/.

[15] US Department of Energy, Office of Energy Efficiency and Renewable Energy 2004. Commercial Unitary Air Conditioners and Heat Pumps Technical Support Document. www.eere.energy.gov/buildings/appliance_standards/commercial/cuac_tsd_060904.html. 


\begin{tabular}{|l|l|l|}
\hline Name & Function & Values \\
\hline Discount Rate & Applied to future dollar savings & percent \\
\hline Program Participation & Fraction of new housing impacted $(\beta)$ & percent \\
\hline $\begin{array}{l}\text { Program Penetration } \\
\text { Scenario }\end{array}$ & Defines the builder decision scenario & $\begin{array}{l}\text { pessimistic,moderate, } \\
\text { optimistic }\end{array}$ \\
\hline $\begin{array}{l}\text { Zero-Cost Efficiency } \\
\text { Improvement }\end{array}$ & Defines savings level for pessimistic scenario & percent \\
\hline $\begin{array}{l}\text { Economic Growth } \\
\text { Scenario }\end{array}$ & Standard AEO projections & low,medium,high \\
\hline $\begin{array}{l}\text { Cost Learning Rate } \\
\text { Fising Energy Price }\end{array}$ & $\begin{array}{l}\text { Defines increased value of savings in optimistic scenario } \\
\text { (see Table 4) }\end{array}$ & percent \\
\hline
\end{tabular}

Table 5: List of input parameters

\section{A BA Model Spreadsheet}

The model described in this report has been implemented in an Excel spreadsheet. In this Appendix we provide a brief description of the model inputs and outputs.

All user inputs and the primary calculation outputs are presented on the Summary worksheet, which is shown in figures 3 and 4. The user inputs are shown in Figure 3, along with two tables that summarize the cumulative impacts over the analysis period (here 2005-2030). The upper table on the worksheet shows the cumulative energy savings, dollar benefits and other quantities by climate zone, while the lower table provides a breakdown of total source energy savings by end-use. Enduse breakdowns are not actually needed in the model, and this table is an approximation based on the assumption that the allocation of total energy to each end-use is constant over time. As detailed data about actual end-use energy use becomes available, this assumption can be modified to provide more accurate estimates. The user inputs visible in Figure 3 are also listed in Table 5, along with their function and the type of values they take. The discount rate is the usual economic discount of future dollar values, and is used to convert the constant dollar net savings to a net present value (NPV). The learning factor $s$ appears in equation ??. The definition of the rising energy price factor is given in Table ??.

Two charts that appear on the Summary worksheet are shown in Figure 4. The first chart shows the annual source energy savings (bars) and number of BA homes built (points). The second chart shows the financial impacts, including the energy cost savings, the additional mortgage cost and the NPV. The ChartNES and ChartNPV worksheets provide charts of the annual energy and cost impacts over the analysis period for each climate zone. The corresponding numerical data is provided on the NES and NPV tabs. Monthly values for the site and source energy savings, and the incremental mortgage costs, can be found on the HouseholdImpacts worksheet.

The Cost Curves worksheet contains all the data used to define the model baseline and the the 
cost curves, as well as the level of source energy savings achievable $(\alpha)$ under all three scenarios. For reference, it also shows the monthly incremental mortgage and energy costs as a function of $\alpha$. For the scenario chosen by the user, the Savings Forecast worksheet provides a table of values for the parameters $\alpha, \alpha_{e}$ and $\alpha_{g}$. The Achievable Savings worksheet contains the BA data used to estimate $\alpha_{e}$ and $\alpha_{g}$, as well as the end-use allocations used to create the table on the Summary page. The Chart Cost Curves worksheet plots the incremental cost curves, and the value of energy savings, as a function of $\alpha$ for each climate zone. The point where the two curves cross is the savings level used in the moderate scenario.

The NewHousingForecast and AEOEnergyPrice worksheets contain these forecasts by census division as taken from the AEO, while the Construction and EnergyPrices worksheets contain the values converted to climate zones. The factors used to do the conversion, defined in equation 1, are listed on the $C D-C Z$ Matrix worksheet.

The model is extremely simple to use - all quantities are immediately recalculated each time the user resets one of the input parameters. 


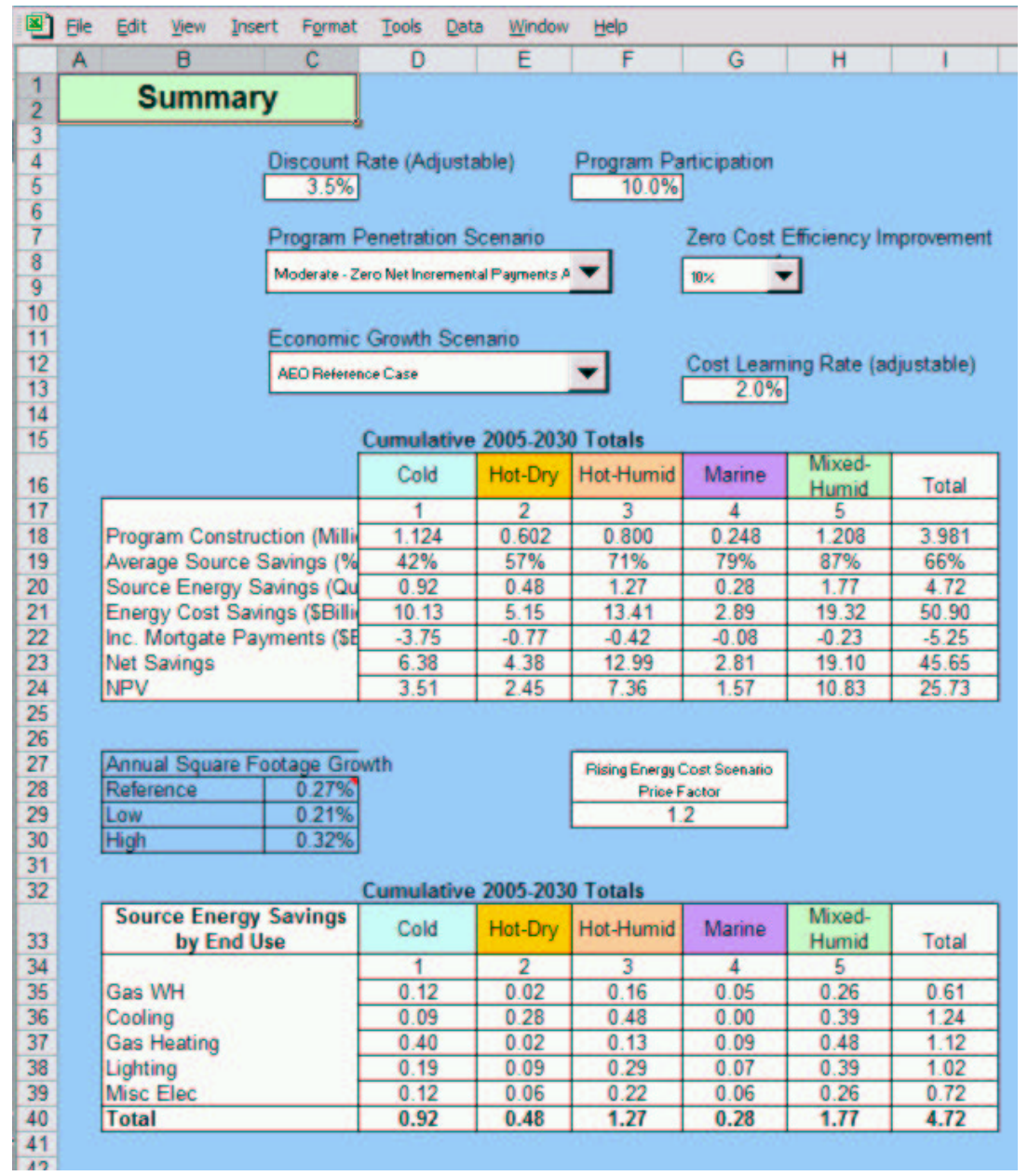

Figure 3: Screen shot of the input selection and summary table area. 


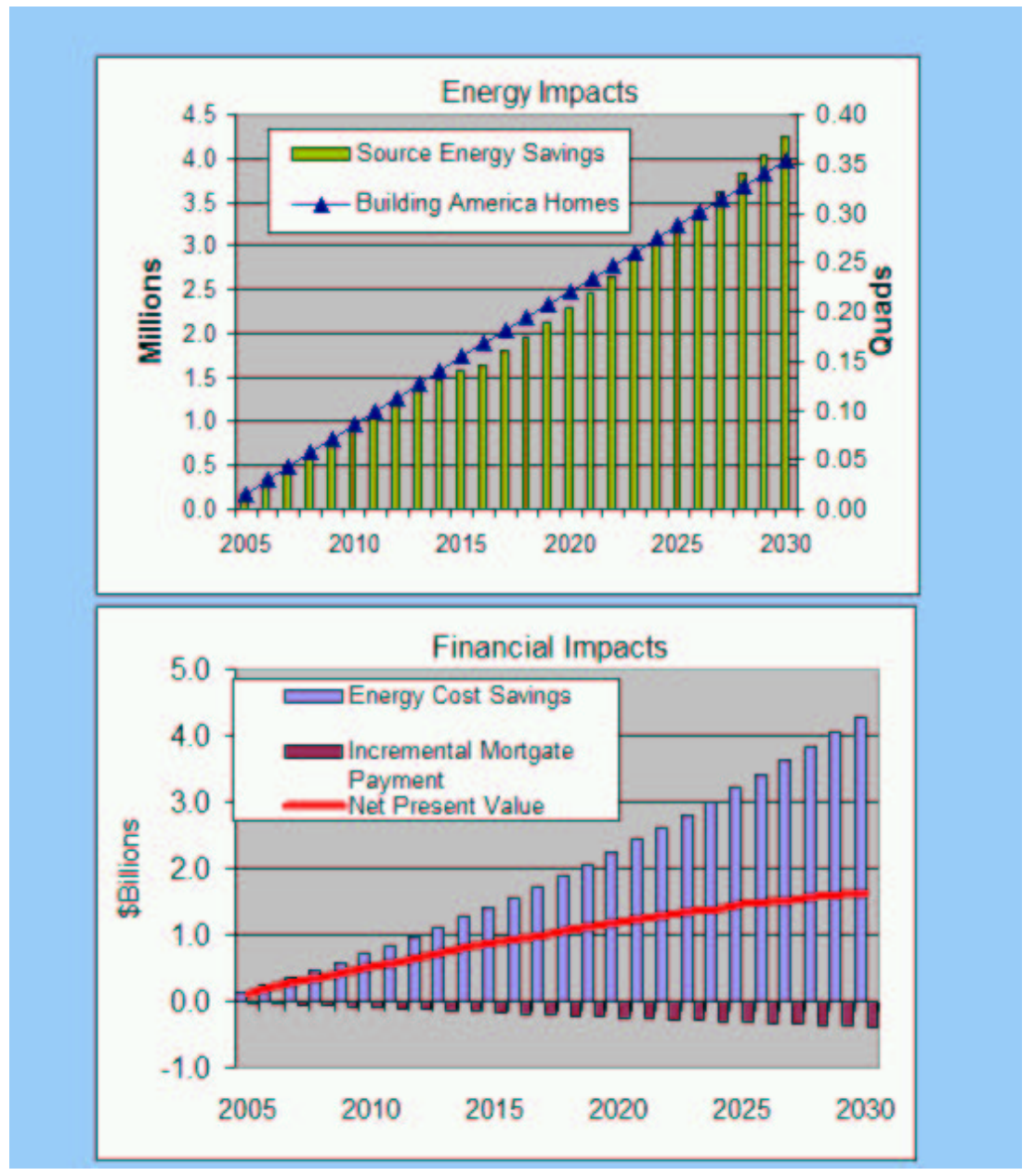

Figure 4: Screen shot of the summary chart area. 